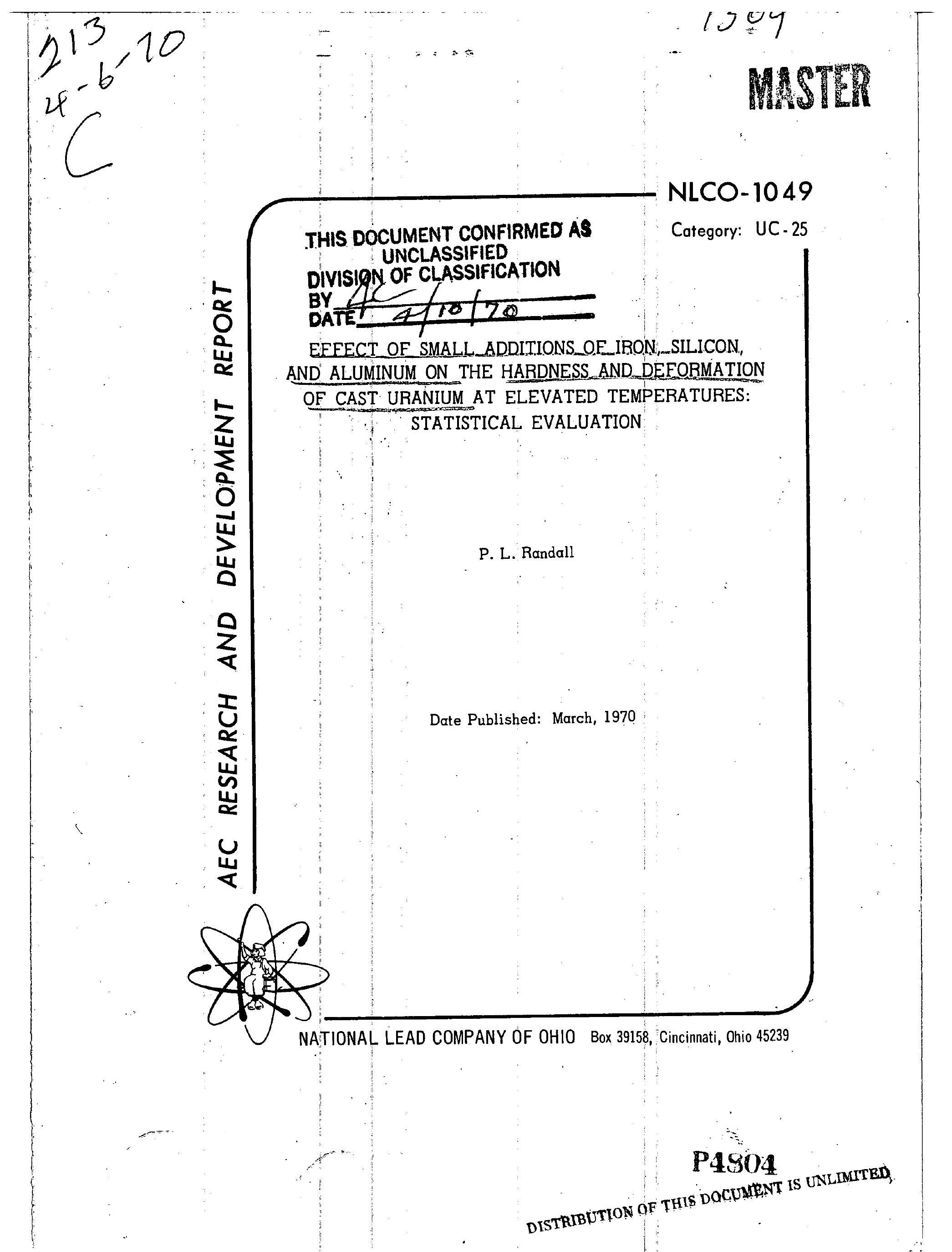


NLC0 $\cdot 1049$

Category: UC.25

\section{EFFECT OF SMALL ADDITIONS OF IRON, SILICON, AND ALUMINUM ON THE HARDNESS AND DEFORMATION OF CAST URANIUM AT ELEVATED TEMPERATURES: STATISTICAL EVALUATION}

Topical Report

P. L. Randall

National Lead Company of Ohio

Technical Division

Box 39158, Cincinnati, Ohio 45239

Prepared for the U.S. Atomic Energy

Commission under Contract No. AT $(30-1) \cdot 1156$

Date Published: March, 1970

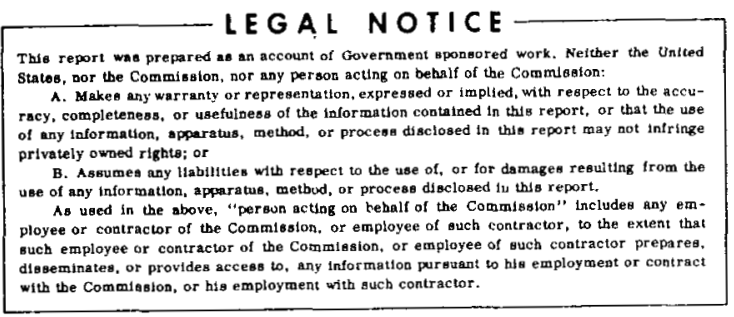

Reproduced in the USA. Available from the

Clearinghouse for Federal Scientific and Technical

Information, Springfield, Virginia 22151.

Price: Full size copy $\$ 3.00$; microfiche copy $\$ .65$.

This document is

PUBLICLY RELEASABLE

B. Stal

Authorizing Official

Date: $\quad 4-17-0 ?$ 


\section{DISCLAIMER}

This report was prepared as an account of work sponsored by an agency of the United States Government. Neither the United States Government nor any agency Thereof, nor any of their employees, makes any warranty, express or implied, or assumes any legal liability or responsibility for the accuracy, completeness, or usefulness of any information, apparatus, product, or process disclosed, or represents that its use would not infringe privately owned rights. Reference herein to any specific commercial product, process, or service by trade name, trademark, manufacturer, or otherwise does not necessarily constitute or imply its endorsement, recommendation, or favoring by the United States Government or any agency thereof. The views and opinions of authors expressed herein do not necessarily state or reflect those of the United States Government or any agency thereof. 


\section{DISCLAIMER}

Portions of this document may be illegible in electronic image products. Images are produced from the best available original document. 


\begin{abstract}
Mechanical property data from 27 cast alloys of the U-Fe- $\mathrm{Si}-\mathrm{Al}$ system were statistically evaluated. The alloy levels were 300 and $1000 \mathrm{ppm}$. Hardness and drop hammer values were obtained at temperatures ranging from 885 to $1200^{\circ} \mathrm{F}$. Both resistance to deformation and hardness values decreased as temperatures increased and increased as the silicon level increased.
\end{abstract}

\title{
INTRODUCTION
}

Small additions of iron and silicon to uranium are known to refine the grains, 1,2 and a finer grain size in uramium, as in most metals, increases the mechanical strength. Higher mechanical strength is being sought because this property in a uranium fuel core apparently restrains swelling during irradiation in the reactor.

In a major investigation of uranium-base dilute alloys, hardness, drop hammer, and tensile tests have been performed on binary, ternary, quaternary, and quinary combinations of uranium with carbon, iron, silicon, and aluminum. ${ }^{3-11}$ To compare the data gathered from the mechanical tests of the various alloy combinations, a statistical analysis of variance was run on the hardness and drop hammer data.

\section{SUMMARY OF RESULTS}

Temperature. The resistance to deformation (inverse of the measured reduction of height) and the hardness of the dilute alloys decreased with increasing test temperature.

Alloying Elements. Silicon was the most influential of the alloying elements in increasing hardness and resistance to deformation. Aluminum also increased hardness and resistance to deformation. Iron had the least effect on the mechanical properties of uranium.

\section{ALLOY PREPARATION}

Specimens for the mechanical property tests were prepared from alloys having a total impurity level of less than $150 \mathrm{ppm}$, except for carbon. Carbon was present in amounts varying from 100 to $900 \mathrm{ppm}$, but based on the results of previous work, carbon is considered to have had little effect on the results. The maximum limit for silicon in alloys without silicon additions was $<30$ ppm, for aluminum in alloys without aluminum additions was $<15 \mathrm{ppm}$, and for iron in alloys without iron additions was $<50 \mathrm{ppm}$.

The 27 alloys were cast under controlled conditions in a laboratory vacuum induction furnace. " Each casting consisted of a cylinder 12 inches long and 3 inches in diameter below which were four integrally cast test bars 7 inches long and $15 / 16$ inch in diameter. These bars were cut from 
the casting body and were radiographed to determine internal soundness. Test specimens were machined from sound portions of the test bars.

Additions of $300 \pm 100 \mathrm{ppm}$ and $1000 \pm 100 \mathrm{ppm}$ were made to produce the required alloys. The average levels of the alloying elements actually found in the various alloys are given in Table I; however, for simplicity, references to these alloys will be by the above nominal composition.

\section{EQUIPMENT}

Drop hammer tests were performed on a machine designed and constructed at this site. Hardness was measured by a standard Rockwell hardness tester modified for elevated temperature testing. Details of the equipment and procedures were reported previously. ${ }^{3,4}$

\section{DESIGN OF THE EXPERIMENT}

All specimens were tested in duplicate at temperatures of $885,955,1025,1095,1150$, and $1200^{\circ} \mathrm{F}$. The factorial block design of the 27 alloys tested is shown in Table I.

The data (Tables II and III) were subjected to statistical analyses of variance and plotted (Figures 1 and 2) to assist in the interpretation of the results and to ascertain the significance of the independent variables $(\mathrm{Fe}, \mathrm{Si}, \mathrm{Al}$, temperature) and their interactions. Duplicate analyses were used to estimate error.

\section{RESULTS}

\section{Drop Hammer Tests}

Specimen deformation was measured by the percent reduction of height. The term "resistance to deformation" (inverse of the measured reduction of height) will be used to describe the drop hammer properties.

Data taken at $1200^{\circ} \mathrm{F}$ were deleted from the statistical analyses because some were anomalous. The anomalies were believed to be caused by the occurrence of a partial alpha-to-beta transformation in these samples during testing. This transformation is possible as tests have demonstrated that the temperature of the specimen increases approximately $50^{\circ} \mathrm{F}$ on impact.

The four-factor analysis of variance showed that ail the independent variables and their interactions were significant at the $99 \%$ confidence level (see Table IV). The results of the threefactor analysis of variance are listed in Table V. Major findings are summarized below:

Effect of Temperature Temperature had a significant effect on resistance to deformation for all alloy combinations ( $99 \%$ confidence level). Resistance to deformation decreased as temperature 
increased (see Table VI). The alloys containing $1000 \mathrm{ppm} \mathrm{Si}$, Al, or Fe were the least affected by temperature changes.

Effect of Iron Level The effect of iron as an additive was significant across all levels of silicon, at the $300 \mathrm{ppm}$ Al level, and at all temperatures studied ( $99 \%$ confidence level). The effect of iron in combination with $1000 \mathrm{ppm}$ Al was not significant (i.e., increasing iron from 50 to 1000 ppm contributed very little to the resistance to deformation when the alloy contained $1000 \mathrm{ppm}$ Al).

Effect of Silicon Level As with temperature, silicon appeared to be most influential on resistance to deformation. The effect of silicon was significant ( $99 \%$ confidence level) at all temperatures tested and at all iron and aluminum levels. Resistance to deformation increased as the silicon level increased (see Table VI).

Effect of Aluminum Level The effect of aluminum as an additive was significant across all levels of silicon, iron, and temperature (99\%) except when iron was at the $1000 \mathrm{ppm}$ level. In this instance, the aluminum level was significant only at $95 \%$. This is to say, the resistance to deformation was affected less by aluminum additions from 15 to $1000 \mathrm{ppm}$ when iron was held at the $1000 \mathrm{ppm}$ level tham when iron was held to less than $1000 \mathrm{ppm}$.

The $1000 \mathrm{ppm} \mathrm{Si}, 1000 \mathrm{ppm} \mathrm{Al}$ alloys had the greatest resistance to deformation of the alloys tested.

\section{Hardness Tests}

The data used in the statistical analysis consisted of the actual experimental data adjusted when replications varied by eight or more Rockwell $\mathrm{H}$ units. Adjustments were needed on only five of the 324 data points and were made using missing value formulas and graphic techniques.

The four-factor analysis of variance showed that each of the independent variables and their interactions was significant (i.e., had a significant effect on the dependent variable, hardness) at the $99 \%$ confidence level with the exception of the two following interactions for which no significant difference was detected: a. Fe $\times$ temperature.

b. Fe $\times \mathrm{Al} \times$ temperature.

The major results are summarized below:

Effect of Temperature Temperature had a significant effect on hardness (99\% confidence level) for all alloy combinations. All alloys became softer as temperatures increased (see Table VI). The $1000 \mathrm{ppm} \mathrm{Si}, 1000 \mathrm{ppm} \mathrm{Al} \mathrm{alloy} \mathrm{(No.} \mathrm{25)} \mathrm{was} \mathrm{the} \mathrm{hardest} \mathrm{and} \mathrm{appeared} \mathrm{to} \mathrm{be} \mathrm{the} \mathrm{least} \mathrm{af-}$ fected by temperature of the 27 alloys.

Effect of Iron Level Iron had a significont effect on hardness (99\% confidence level) at all levels of aluminum. However, the iron level had no significant effect with silicon at the $1000 \mathrm{ppm}$ 
level. Iron had a significant effect on hardness (99\%) at all temperature levels except 1095 and $1200^{\circ} \mathrm{F}$. Additions of iron from 50 to $300 \mathrm{ppm}$ sometimes caused a softening of the alloy (see Table VI).

Effect of Silicon Level Along with temperature, silicon appeared to be most influential on hardness. The effect of silicon was significant ( $99 \%$ confidence level) at all temperatures tested and at all iron and aluminum levels. Hardness increased as the silicon level increased (see Table VI).

Effect of Aluminum Level The aluminum level was significant ( $99 \%$ confidence level) at all levels of iron and silicon and at all temperatures tested. Increasing the level of aluminum resulted in increased hardness (see Table VI). However, the F ratios seem to indicate that aluminum had a greater effect on hardness when silicon was not present as an additive element.

\section{REFERENCES}

1P. H. Dixon, F. H. Fern, and B. R. Butcher. The Solution of Precipitates in Very Dilute U-Fe-Al Alloys, UKAEA Report AERE-R-4636. May 1964.

${ }^{2}$. R. Kaufmann. Nuclear Reactor Fuel Elements; Metallurgy and Fabrication, p. 72. New York: Interscience, 1962.

3L. G. Wexlin and R. E. DuVall. "Elevated-Temperature Hardness of Uranium-Base Dilute Binary Alloys," Summary Technical Report for the Period October 1, 1966 to December 31, 1966, USAEC Report NLCO-995, pp. 4.1-4.4. January, 1967.

4L. G. Wexlin and R. E. DuVall. "Elevated-Temperature Drop-Hammer Tests of Cast Uranium-Base Dilute Binary Alloys," Summary Technical Report for the Period January 1, 1967 to March 31, 1967, USAEC Report NLCO-996, pp. 2.1-2.6. April, 1967.

5L. G. Wexlin and R. E. DuVall. "Elevated-Temperature Drop-Hammer and Hardness Tests of Cast Uranium-Base Dilute Ternary Alloys," Summary Technical Report for the Period October 1, 1967 to December 31, 1967, USAEC Report NLCO - 1010, pp. 2.1-2.6. February, 1968.

${ }^{6}$ L. G. Wexlin and R. E. DuVall. "Elevated-Temperature Tensile, Drop Hammer, and Hardness Tests of Cast Uranium-Base Dilute Quaternary Alloys," Summary Technical Report for the Period January 1, 1968 to March 31, 1968, USAEC Report NLCO-1015, pp. 3.1 - 3.11. May, 1968.

7P. L. Randall, L. G. Wexlin, and R. E. DuVall. "Elevated-Temperature Drop Hammer and Hardness Tests of Cast Uranium-Base Dilute Quinary Alloys," Summary Technical Report for the Period July 1, 1968 to September 30, 1968, USAEC Report NLCO-1025, pp. 4.1-4.8. November, 1968.

8L. G. Wexlin. "Elevated-Temperature Tensile Tests of Cast Uranium-Base Dilute Binary Alloys," Summary Technical Report for the Period April 1, 1967 to June 30, 1967, USAEC Report NLCO-1001, pp. 5.1 - 5.9. August, 1967.

${ }^{9}$ L. G. Wexlin. "Elevated-Temperature Tensile Tests of Cast Uranium-Base Dilute Ternary Alloys," Summary Technical Report for the Period July 1, 1967 to September 30, 1967, USAEC Report NLCO-1005, pp. 5.1 - 5.10. November, 1967.

10 L. G. Wexlin and E. L. Schaich. Microstructures of As-Cast Uranium-Base Dilute Alloys, USAEC Report NLCO-1022. February, 1969.

$11_{G}$. Brodi. "Techniques for Producing Uranium Castings of Complex Configuration for Physical Testing," Summary Technical Report for the Period July 1, 1963 to September 30, 1963, USAEC Report NLCO-890, pp. $17-20$. October 28, 1963. 
TABLE I Factorial Block Design - 27 Alloys of the U-Fe -Si - Al System*

\begin{tabular}{|c|c|c|c|c|c|c|c|c|c|}
\hline & \multicolumn{3}{|c|}{$<30 \mathrm{ppm} \mathrm{Si}$} & \multicolumn{3}{|c|}{$300 \mathrm{ppm} \mathrm{Si}$} & \multicolumn{3}{|c|}{$1000 \mathrm{ppm} \mathrm{Si}$} \\
\hline & $\begin{array}{c}<15 \mathrm{ppm} \\
\mathrm{Al}\end{array}$ & $\begin{array}{c}300 \mathrm{ppm} \\
\mathrm{Al}\end{array}$ & $\begin{array}{c}1000 \mathrm{ppm} \\
\mathrm{Al}\end{array}$ & $\begin{array}{c}<15 \mathrm{ppm} \\
\mathrm{Al}\end{array}$ & $\begin{array}{c}300 \mathrm{ppm} \\
\mathrm{Al}\end{array}$ & $\begin{array}{c}1000 \mathrm{ppm} \\
\mathrm{Al}\end{array}$ & $\begin{array}{c}<15 \mathrm{ppm} \\
\mathrm{Al}\end{array}$ & $\begin{array}{c}300 \mathrm{ppm} \\
\text { Al }\end{array}$ & $\begin{array}{c}1000 \mathrm{ppm} \\
\mathrm{Al}\end{array}$ \\
\hline$<50$ ppm $\mathrm{Fe}$ & 1 & 4 & 7 & 10 & 13 & 16 & 19 & 22 & 25 \\
\hline $300 \mathrm{ppm} \mathrm{Fe}$ & 2 & 5 & 8 & 11 & 14 & 17 & 20 & 23 & 26 \\
\hline $1000 \mathrm{ppm} \mathrm{Fe}$ & 3 & 6 & 9 & 12 & 15 & 18 & 21 & 24 & 27 \\
\hline
\end{tabular}

* Numbers in blocks are alloy designations.

TABLE II Average Reduction of Height (\%) of Uranium-Base Dilute Alloys

\begin{tabular}{|c|c|c|c|c|c|c|c|c|c|c|}
\hline \multirow[t]{2}{*}{$\begin{array}{c}\text { Alloy } \\
\text { No. }\end{array}$} & \multicolumn{3}{|c|}{$\begin{array}{c}\text { Average Alloy } \\
\text { Composition (ppm) }\end{array}$} & \multicolumn{6}{|c|}{ Test Temperature $\left({ }^{\circ} \mathrm{F}\right)$} & \multirow{2}{*}{$\begin{array}{l}\text { Change in } \\
\text { Reduction } \\
\text { of Height * }\end{array}$} \\
\hline & $\mathrm{Fe}$ - & $\mathrm{Si}_{\mathrm{i}}$ & Al & 885 & 955 & 1025 & 1095 & 1150 & 1200 & \\
\hline 1 & - & - & - & 29.4 & 32.5 & 30.2 & 35.6 & 38.6 & 36.1 & 9.2 \\
\hline 2 & 340 & - & - & 26.6 & 30.4 & 34.2 & 37.8 & 40.0 & 40.7 & 13.4 \\
\hline 3 & 970 & - & - & 27.3 & 29.2 & 31.2 & 29.4 & 36.0 & 30.8 & 8.7 \\
\hline 4 & - & - & 330 & 27.8 & 30.5 & 33.6 & 35.4 & 39.4 & 34.5 & 11.6 \\
\hline 5 & 230 & - & 230 & 27.8 & 26.8 & 34.1 & 37.0 & 39.4 & 37.8 & 11.6 \\
\hline 6 & 1000 & - & 250 & 23.6 & 27.5 & 30.2 & 31.1 & 32.4 & 35.1 & 8.8 \\
\hline 7 & - & - & 1040 & 23.4 & 26.4 & 29.8 & 30.4 & 36.1 & 29.1 & 12.7 \\
\hline 8 & 230 & - & 980 & 22.9 & 24.8 & 27.5 & 31.0 & 32.8 & 32.8 & 9.9 \\
\hline 9 & 1000 & - & 1050 & 22.6 & 24.1 & 28.5 & 31.6 & 34.5 & 34.5 & 11.9 \\
\hline 10 & - & 340 & - & 24.6 & 28.6 & 32.0 & 35.4 & 37.3 & 32.8 & 12.7 \\
\hline 11 & 230 & 240 & - & 23.4 & 17.0 & 30.1 & 33.8 & 37.2 & 36.5 & 13.8 \\
\hline 12 & 1020 & 210 & - & 14.4 & 22.4 & 24.4 & 31.6 & 35.2 & 33.2 & 20.8 \\
\hline 13 & - & 230 & 230 & 24.7 & 27.6 & 31.4 & 34.6 & 34.6 & 35.2 & 9.9 \\
\hline 14 & 220 & 200 & 240 & . 25.4 & 29.8 & 31.5 & 34.6 & 39.4 & 36.1 & 14.0 \\
\hline 15 & 940 & 250 & 260 & 21.5 & 24.8 & 25.8 & 30.1 & 33.6 & 28.8 & 12.1 \\
\hline 16 & - & 230 & 1000 & 21.5 & 23.8 & 28.0 & 28.4 & 31.0 & 32.4 & 9.5 \\
\hline 17 & 250 & 280 & 1020 & 22.0 & 23.9 & 26.6 & 27.4 & 32.1 & 32.5 & 10.1 \\
\hline 18 & 950 & 190 & 990 & 22.4 & 22.6 & 27.6 & 30.2 & 33.0 & 34.9 & 10.6 \\
\hline 19 & - & 990 & - & 21.7 & 23.4 & 25.9 & 31.0 & 30.0 & 28.8 & 8.3 \\
\hline 20 & 240 & 940 & - & 18.6 & 15.4 & 23.4 & 26.5 & 27.2 & 29.3 & 8.6 \\
\hline 21 & 1020 & 960 & - & 18.7 & 22.2 & 23.0 & 24.6 & 29.3 & 30.3 & 10.6 \\
\hline 22 & - & 940 & 220. & 17.8 & 20.0 & 24.4 & 25.0 & 27.4 & 26.3 & 9.6 \\
\hline 23 & 260 & 1070 & 250 & 18.5 & 21.8 & 23.8 & 25.9 & 29.9 & 31.7 & 11.4 \\
\hline 24 & 960 & 930 & 220 & 17.7 & 19.8 & 22.1 & 24.1 & 26.4 & 28.7 & 8.7 \\
\hline 25 & - & 910 & 960. & 18.8 & 19.0 & 21.3 & 23.8 & 24.8 & 26.1 & 6.0 \\
\hline 26 & 240 & 990 & 980 & 18.4 & 20.8 & 22.4 & 24.1 & 28.6 & 30.0 & 10.2 \\
\hline 27 & 1020 & 930 & 970 & 17.2 & 19.0 & 21.2 & 25.1 & 27.8 & 29.8 & 10.6 \\
\hline
\end{tabular}

* Data at $1200^{\circ} \mathrm{F}$ not included

95\% Confidence limits on each mean value listed $= \pm 0.19$ 
TABLE III Average Hardness $\left(\mathrm{R}_{\mathrm{H}}\right)$ of

Uranium - Base Dilute Alloys

\begin{tabular}{|c|c|c|c|c|c|c|c|}
\hline \multirow{2}{*}{$\begin{array}{l}\text { Alloy } \\
\text { No. }\end{array}$} & \multicolumn{6}{|c|}{ Test Temperature $\left({ }^{\circ} \mathrm{F}\right)$} & \multirow{2}{*}{$\begin{array}{l}\text { Change in } \\
\text { Hardness }\end{array}$} \\
\hline & 885 & 955 & 1025 & 1095 & 1150 & 1200 & \\
\hline 1 & 99 & 99 & 93 & 90 & 79 & 75 & 24 \\
\hline 2 & 100 & 96 & 88 & 84 & 64 & 60 & 40 \\
\hline 3 & 103 & 100 & 96 & 90 & 74 & 66 & 37 \\
\hline 4 & 106 & 105 & 96 & 91 & 84 & 76 & 30 \\
\hline 5 & 104 & 102 & 97 & 92 & 86 & 81 & 23 \\
\hline 6 & 109 & 106 & 104 & 96 & 92 & 84 & 25 \\
\hline 7 & 108 & 106 & 104 & 102 & 94 & 88 & 20 \\
\hline 8 & 111 & 106 & 106 & 99 & 98 & 96 & 15 \\
\hline 9 & 110 & 105 & 102 & 95 & 93 & 86 & 24 \\
\hline 10 & 110 & 106 & 102 & 98 & 90 & 84 & 26 \\
\hline 11 & 104 & 102 & 98 & 91 & 89 & 82 & 22 \\
\hline 12 & 109 & 108 & 104 & 100 & 94 & 90 & 19 \\
\hline 13 & 104 & 104 & 103 & 101 & 96 & 88 & 16 \\
\hline 14 & 104 & 104 & 99 & 98 & 91 & 86 & 18 \\
\hline 15 & 110 & 110 & 108 & 103 & 96 & 86 & 24 \\
\hline 16 & 112 & 110 & 108 & 105 & 99 & 98 & 14 \\
\hline 17 & 110 & 109 & 106 & 108 & 98 & 92 & 18 \\
\hline 18 & 110 & 108 & 108 & 104 & 97 & 94 & 16 \\
\hline 19 & 114 & 112 & 109 & 104 & 100 & 92 & 22 \\
\hline 20 & 114 & 111 & 111 & 107 & 102 & 97 & 17 \\
\hline 21 & 114 & 112 & 110 & 104 & 100 & 97 & 17 \\
\hline 22 & 113 & 112 & 110 & 106 & 104 & 96 & 17 \\
\hline 23 & 114 & 112 & 110 & 106 & 103 & 96 & 18 \\
\hline 24 & 114 & 114 & 112 & 107 & 102 & 98 & 16 \\
\hline 25 & 115 & 113 & 112 & 108 & 106 & 102 & 13 \\
\hline 26 & 114 & 114 & 112 & 110 & 104 & 100 & 14 \\
\hline 27 & 114 & 113 & 113 & 107 & 103 & 100 & 14 \\
\hline
\end{tabular}

$95 \%$ Confidence limits on each mean value listed $= \pm 0.22$. 
TABLE IV Four-Factor Analysis of Variance of Drop Hammer and Hardness Data

\begin{tabular}{|c|c|c|c|c|c|}
\hline \multirow{3}{*}{$\begin{array}{l}\text { Variables } \\
\text { and } \\
\text { Interactions }\end{array}$} & \multicolumn{2}{|c|}{ Drop Hammer } & \multicolumn{3}{|c|}{ Hardness } \\
\hline & \multirow[t]{2}{*}{$\begin{array}{l}\text { Computed } \\
\text { F Ratio }\end{array}$} & \multirow[t]{2}{*}{$\begin{array}{c}\text { F Level to be } \\
\text { Exceeded at } \\
99 \% \text { Confidence } \\
\text { Level }\end{array}$} & \multirow[t]{2}{*}{$\begin{array}{l}\text { Computed } \\
\text { F Ratio }\end{array}$} & \multicolumn{2}{|c|}{$\begin{array}{c}\text { F Level to be } \\
\text { Exceeded at } \\
\text { Indicated } \\
\text { Confidence Level }\end{array}$} \\
\hline & & & & $95 \%$ & $99 \%$ \\
\hline Iron & 93.3 & 4.77 & 52.0 & - & 4.74 \\
\hline Silicon & 1121.3 & 4.77 & 2470.6 & - & 4.74 \\
\hline Aluminum & 150.0 & 4.77 & 845.4 & - & 4.74 \\
\hline Temperature & 812.8 & 3.46 & 1781.9 & - & 3.13 \\
\hline $\mathrm{Fe}_{\mathrm{e}} \times \mathrm{Si}_{\mathrm{i}}$ & 8.2 & 3.46 & 19.0 & - & 3.44 \\
\hline $\mathrm{Fe} \times \mathrm{Si}_{i}$ & 39.7 & 3.46 & 54.2 & - & 3.44 \\
\hline $\mathrm{Fe} \times \mathrm{Temp}$ & 6.2 & 2.64 & $1.83^{\star}$ & 1.89 & 2.44 \\
\hline $\mathrm{Si} \times \mathrm{Al}$ & 22.4 & 3.46 & 167.0 & - & 3.44 \\
\hline $\mathrm{S}_{\mathrm{i}} \times \mathrm{Temp}$ & 7.0 & 2.64 & 42.0 & - & 2.44 \\
\hline $\mathrm{Al} \times \mathrm{Temp}$ & 3.69 & 2.64 & 30.8 & - & 2.44 \\
\hline $\mathrm{Fe} \times \mathrm{Si} \times \mathrm{Al}$ & 12.6 & 2.64 & 21.6 & - & 2.62 \\
\hline $\mathrm{Fe} \times \mathrm{S}_{\mathrm{i}} \times \mathrm{Temp}$ & 3.66 & 2.14 & 2.06 & - & 2.00 \\
\hline $\mathrm{Fe} \times \mathrm{Al}_{1} \times \mathrm{Temp}$ & 6.8 & 2.14 & $1.30 *$ & 1.64 & 2.00 \\
\hline $\mathrm{Si} \times \mathrm{Al}_{\mathrm{I}} \times \mathrm{Temp}$ & 6.0 & 2.14 & 9.24 & - & 2.00 \\
\hline $\begin{array}{l}\overline{\mathrm{Fe}} \times \mathrm{Si} \times \\
\mathrm{Al} \times \mathrm{Temp}\end{array}$ & 3.04 & 1.82 & 6.64 & - & 1.72 \\
\hline
\end{tabular}

- Not significant 
TABLE V Three-Factor Analysis of Variance of Drop Hammer Data, F Ratios

\begin{tabular}{|c|c|c|c|c|c|c|c|c|c|c|c|}
\hline \multirow[t]{3}{*}{. } & \multicolumn{9}{|c|}{ Elements and Levels } & \multirow{2}{*}{\multicolumn{2}{|c|}{$\begin{array}{l}\text { F Level to } \\
\text { be Exceeded }\end{array}$}} \\
\hline & \multicolumn{3}{|c|}{ Aluminum } & \multicolumn{3}{|c|}{ Silicon } & \multicolumn{3}{|c|}{ Iron } & & \\
\hline & 1 & 2 & 3 & 1 & 2 & 3 & 1 & 2 & 3 & $95 \%$ & $99 \%$ \\
\hline Temperature & 193.96 & 402.07 & 356.92 & 227.63 & 431.89 & 197.61 & 238.02 & 271.42 & 320.79 & 2.58 & 3.77 \\
\hline Iron & 50.27 & 135.20 & N.S. & 43.53 & 63.98 & 6.32 & & & & 3.19 & 5.11 \\
\hline Silicon & 244.73 & 786.35 & 377.79 & & & & 461.49 & 363.91 & 312.03 & 3.19 & 5.11 \\
\hline Aluminum & & & & 100.62 & 72.05 & 20.69 & 141.10 & 78.07 & $4.02^{\star}$ & 3.19 & 5.11 \\
\hline$T e m p \times F e$ & 8.95 & 3.66 & 3.16 & 4.07 & 7.88 & $2.16^{\star}$ & & & & 2.15 & 2.93 \\
\hline$\overline{\mathrm{Temp}} \times \mathrm{Si}$ & 9.07 & 2.96 & $2.60 *$ & & & & 4.46 & $2.31^{\star}$ & 8.66 & 2.15 & 2.93 \\
\hline Temp $\times A l$ & & & & N.S. & 13.73 & N.S. & $2.75^{\star}$ & 8.79 & 4.67 & 2.15 & 2.93 \\
\hline $\mathrm{Fe}_{\mathrm{e}} \times \mathrm{S}_{\mathrm{i}}$ & 12.01 & 13.25 & 6.83 & & & & & & & 2.58 & 3.77 \\
\hline $\mathrm{Fe} \times \mathrm{Al}$ & & & & 11.15 & 38.50 & 18.98 & & & & 2.58 & 3.77 \\
\hline$\overline{\mathrm{Si} \times \mathrm{Al}}$ & & & & & & & 8.38 & 24.40 & 11.99 & 2.58 & 3.77 \\
\hline $\mathrm{Temp} \times \mathrm{Fe} \times \mathrm{Si}$ & 3.72 & 3.44 & N.S. & & & & & & & 1.88 & $\overline{2.43}$ \\
\hline Temp $\times \mathrm{Fe} \times \mathrm{Al}$ & & & & 3.40 & 7.15 & 2.87 & & & & 1.88 & 2.43 \\
\hline \multirow[t]{3}{*}{ Temp $\times \mathrm{Si} \times \mathrm{Al}$} & & & & & & & $1.95^{\star}$ & 4.35 & 5.90 & 1.88 & 2.43 \\
\hline & \multicolumn{9}{|c|}{ Temperature Levels } & & \\
\hline & 1 & 2 & 3 & 4 & 5 & & & & & & \\
\hline Iron & 30.63 & 25.04 & 22.71 & 20.16 & 20.23 & & & & & 3.35 & 5.49 \\
\hline Silicon & 193.49 & 226.97 & 199.41 & 186.68 & 402.04 & & & & & 3.35 & 5.49 \\
\hline Aluminum & 15.01 & 27.71 & 25.72 & 42.81 & 57.38 & & & & & 3.35 & 5.49 \\
\hline $\mathrm{Fe} \times \mathrm{Si}_{i}$ & 4.90 & $2.99^{\star}$ & $3.58^{\star}$ & 4.13 & 8.63 & & & & & 2.73 & 4.11 \\
\hline $\mathrm{Fe} \times \mathrm{Al}$ & 8.24 & 23.27 & 5.17 & 14.94 & 17.14 & & & & & 2.73 & 4.11 \\
\hline $\mathrm{Si}_{\mathrm{i}} \times \mathrm{Al}$ & 16.43 & 21.69 & N.S. & 4.22 & 4.62 & & & & & 2.73 & 4.11 \\
\hline $\mathrm{Fe} \times \mathrm{Si} \times \mathrm{Al}$ & 6.34 & 8.41 & 3.85 & N.S. & 5.63 & & & & & 2.30 & 3.26 \\
\hline
\end{tabular}

* Significant at $95 \%$ confidence leve 1

N.S. - Not Significant 
TABLE VI Average Value of Percent Reduction of Height and Hardness at Each Level of the Independent Variables

\begin{tabular}{|c|c|c|c|}
\hline & $\begin{array}{l}\text { Variable } \\
\text { (ppm) }\end{array}$ & $\begin{array}{c}\text { Average } \% \\
\text { Reduction of Height }\end{array}$ & $\begin{array}{l}\text { Average } \\
\text { Hardness }\end{array}$ \\
\hline \multirow{4}{*}{ 号 } & & $( \pm .24)^{\star}$ & $( \pm .27)^{\star}$ \\
\hline & $<50$ & 28.37 & 100.69 \\
\hline & 300 & 27.84 & 99.58 \\
\hline & 1000 & 26.15 & 101.53 \\
\hline \multirow{3}{*}{ 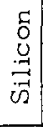 } & $<30$ & 30.91 & 93.93 \\
\hline & 300 & 28.39 & 100.50 \\
\hline & 1000 & 23.06 & 107.38 \\
\hline \multirow{3}{*}{ 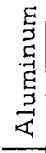 } & $<15$ & 28.37 & 96.66 \\
\hline & 300 & 28.23 & 100.61 \\
\hline & 1000 & 25.76 & 104.52 \\
\hline \multirow{2}{*}{$\begin{array}{l}2 \\
\text { O }\end{array}$} & & $( \pm .31)^{*} \bullet$ & $( \pm .38)^{*}$ \\
\hline & 885 & 22.19 & 109.30 \\
\hline \multirow{5}{*}{$\begin{array}{l}0 \\
0 \\
5 \\
0 \\
0 \\
0 \\
0 \\
\Xi \\
0 \\
E-1\end{array}$} & 955 & 24.22 & 107.40 \\
\hline & 1025 & 27.57 & 104.31 \\
\hline & 1095 & 30.19 & 100.11 \\
\hline & 1150 & 33.10 & 94.00 \\
\hline & 1200 & - & 88.48 \\
\hline
\end{tabular}

* $95 \%$ Confidence limits on means listed for $\mathrm{Al}, \mathrm{Si}$ and $\mathrm{Fe}$ * $95 \%$ Confidence limits on means listed for Temperature 
TABLE VII Three - Factor Analysis of Variance of Hardness Data, F Ratios

\begin{tabular}{|c|c|c|c|c|c|c|c|c|c|c|c|}
\hline & \multicolumn{11}{|c|}{ Elements and Levels } \\
\hline & \multicolumn{3}{|c|}{ Aluminum } & \multicolumn{3}{|c|}{ Silicon } & \multicolumn{3}{|c|}{ Iron } & \multicolumn{2}{|c|}{$\begin{array}{c}\text { F Level to } \\
\text { be Exceeded }\end{array}$} \\
\hline & 1 & 2 & 3 & 1 & 2 & 3 & 1 & 2 & 3 & $95 \%$ & $99 \%$ \\
\hline Temperature & 801.38 & 492.38 & 526.40 & 1104.84 & 364.24 & 528.63 & 562.00 & 700.45 & 547.94 & & 3.38 \\
\hline Iron & 64.62 & 61.48 & 21.99 & 25.74 & 46.05 & N.S. & & & & 3.17 & 5.02 \\
\hline Silicon & 1618.94 & 568.19 & 474.28 & & & & 748.83 & 1229.38 & 628.35 & & 5.02 \\
\hline Aluminum & & & & 1059.38 & 139.00 & 58.51 & 276.56 & 626.75 & 131.33 & & 5.02 \\
\hline Temp $\times \mathrm{Fe}$ & N.S. & N.S. & $2.14 *$ & 4.30 & N.S. & N.S. & & & & 2.00 & 2.67 \\
\hline Temp $\times S_{i}$ & 38.09 & 10.80 & 7.65 & & & & 11.50 & 20.21 & 15.22 & & 2.67 \\
\hline Temp $\times A 1$ & & & & 43.24 & 5.72 & 3.98 & 9.07 & 19.64 & 6.81 & & 2.67 \\
\hline $\mathrm{Fe} \times \mathrm{S}_{\mathrm{i}}$ & 37.15 & 10.95 & 11.38 & & & & & & & & 3.69 \\
\hline $\mathrm{Fe} \times \mathrm{Al}$ & & & & 82.47 & 14.22 & 5.71 & & & & & 3.69 \\
\hline$S_{i} \times A l$ & & & & & & & 21.75 & 165.26 & 42.95 & & 3.69 \\
\hline Temp $\times F_{e} \times S_{i}$ & 7.14 & 2.79 & 5.87 & & & & & & & & 2.23 \\
\hline Temp $\times \mathrm{Fe} \times \mathrm{Al}$ & & & & 11.00 & 2.46 & $1.89^{*}$ & & & & 1.76 & 2.23 \\
\hline \multirow[t]{3}{*}{ Temp $\times$ Si $\times A L$} & & & & & & & 3.82 & 14.70 & 5.49 & & 2.23 \\
\hline & \multicolumn{9}{|c|}{ Temperature Levels } & & \\
\hline & 1 & 2 & 3 & 4 & 5 & 6 & & & & & \\
\hline Iron & 13.98 & 13.18 & 16.56 & $4.66^{\star}$ & 10.50 & N.S. & & & & 3.35 & 5.49 \\
\hline Silicon & 217.77 & 379.37 & 260.26 & 405.08 & 691.89 & 667.65 & & & & & 5.49 \\
\hline Aluminum & 55.33 & 68.53 & 72.49 & 145.66 & 274.84 & 321.32 & & & & & 5.49 \\
\hline $\mathrm{Fe} \times \mathrm{S}_{i}$ & 4.62 & 4.27 & $3.13^{*}$ & 7.04 & 5.64 & 4.88 & & & & 2.73 & 4.11 \\
\hline $\mathrm{Fe} \times \mathrm{Al}_{\mathrm{l}}$ & 5.12 & 10.54 & 6.65 & 14.22 & 12.46 & 11.58 & & & & & 4.11 \\
\hline $\mathrm{S}_{1} \times \mathrm{Al}_{1}$ & 23.02 & 17.39 & 13.06 & 12.22 & 80.00 & 59.65 & & & & & 4.11 \\
\hline $\mathrm{Fe} \times \mathrm{Si} \times \mathrm{Al}$ & 3.90 & N.S. & 3.69 & 4.96 & 14.24 & 22.10 & & & & 2.30 & 3.26 \\
\hline
\end{tabular}

* Significant at $95 \%$ confidence level

N.S. - Not Significant 


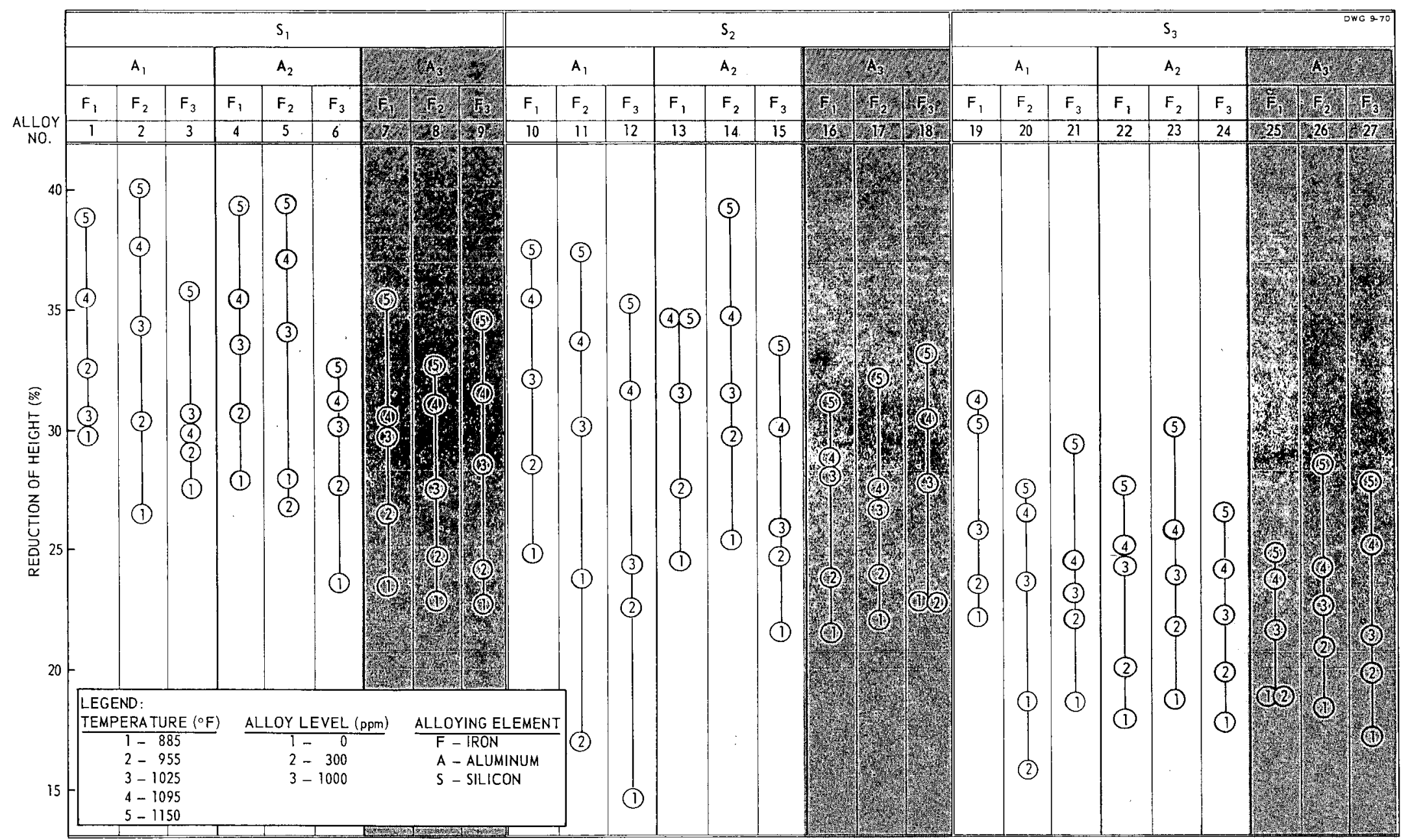

FIGURE 1 Drop Hammer Data 


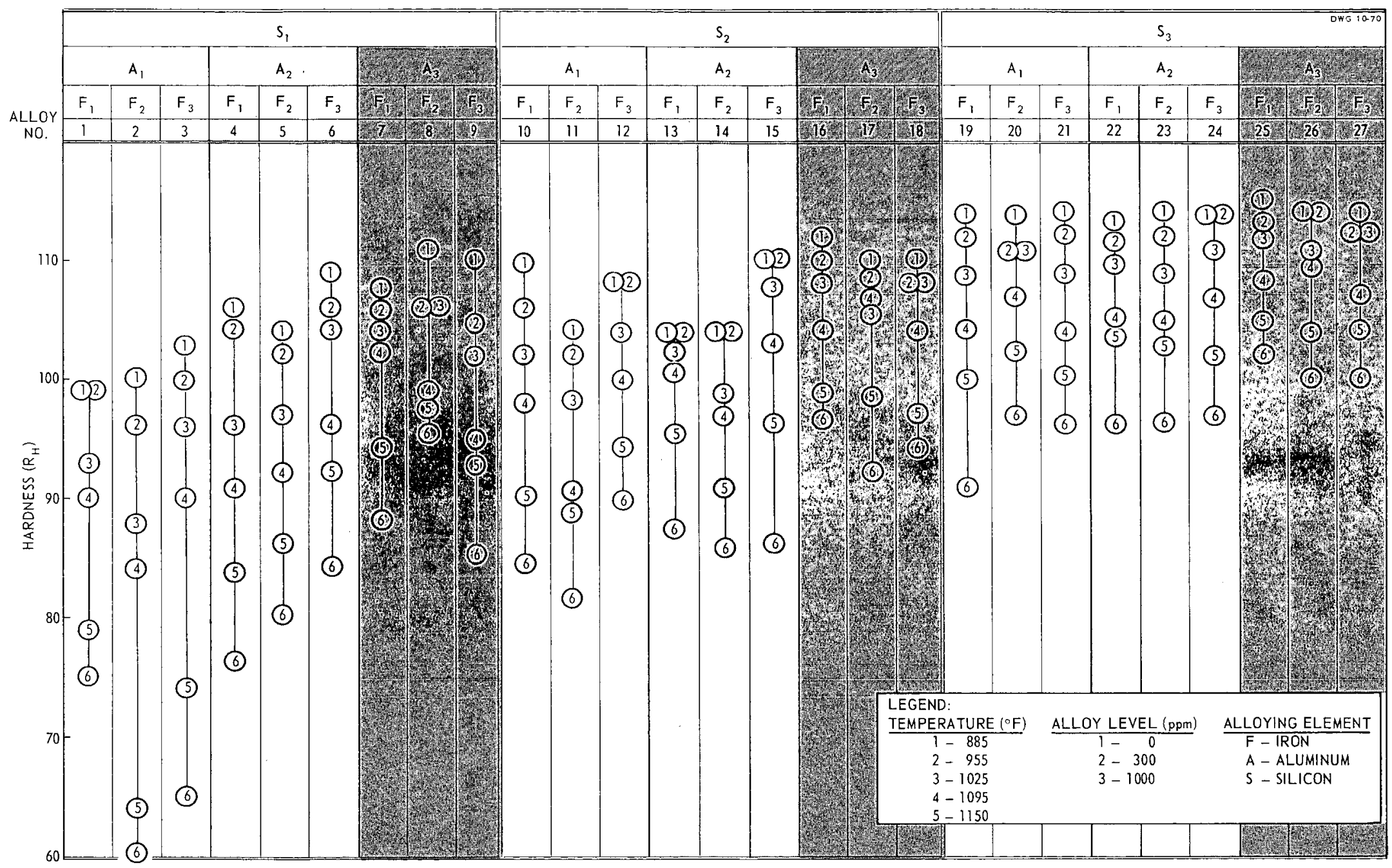

FIGURE 2 Hardness Data 